AN INVERSE PROBLEM, WITH BOUNDARY

MEASUREMENTS FOR THE STEADY STATE DIFFUSION EQUATION

T.J. Connolly and David J.N. Wall

No. 40

October 1987 


\title{
AN INVERSE PROBLEM, WITH BOUNDARY MEASUREMENTS FOR THE STEADY STATE DIFFUSION EQUATION
}

\begin{abstract}
The Newton-Kantorovich computational method is applied to the inverse problem of reconstructing a conductivity from given boundary measurements. The paper provides the theoretical analysis necessary to provide rigorous derivations of the Fréchet differential. Linearisations of the non-linear problem are examined and a numerical procedure for reconstructing general conductivities suggested. This procedure is illustrated by reconstructing one-dimensional conductivities.
\end{abstract}

\section{$1 \quad$ Introduction}

The classical direct problem associated with the partial differential equation

$$
\nabla \cdot[f(\underline{x}) \nabla u(\underline{x})]=0, \quad \underline{x} \in \mathbf{R}^{n}, \quad n=2 \text { or } 3,
$$

is to determine $u$, given $f$ and appropriate boundary conditions on the boundary $\partial \Omega$ of the bounded, simply connected open region $\Omega$. We shall assume the boundary $\partial \Omega$ is a $C^{1}$ mapping of a compact interval $I \subset \mathbf{R}$ into $\mathbf{R}^{n}$. The boundary conditions we shall consider are either Dirichlet

$$
u(\underline{x})=g(\underline{x}), \quad \underline{x} \in \partial \Omega,
$$

or the normalised Neumann boundary condition

$$
f(\underline{x}) \frac{\partial u}{\partial \nu}=g(\underline{x}), \quad \underline{x} \in \partial \Omega,
$$

where $\partial / \partial \nu$ denotes the directional derivative in the direction of the unit outward normal vector $\underline{\hat{v}}$ to $\partial \Omega$.

The inverse or identification problem we shall examine in this paper, is to determine the spatially varying function $f$ from knowledge of $u(\underline{x})$ for $\underline{x} \in \partial \Omega$; that is certain measurements of $u$ on the boundary. These measurements consist of:

(a). If Dirichlet conditions are specified on $\partial \Omega$ - then $f \partial u / \partial \nu$ is measured on $\partial \Omega$.

(b). If Neumann conditions are specified on $\partial \Omega$ - then $u$ is measured on $\partial \Omega$.

Of course these are not the only permissible measurements, but a necessary condition for this problem is that the measurement set must be independent of the specified set.

The inverse problem for equation (1.1) is of considerable importance as it arises from several physical problems. A major application is in electrical conductivity imaging, or "impedance tomography" as the medical technique of non-invasive conductivity measurement is called (see [1], [2], [3]). It also arises in two geophysical problems. The first is in geophysical prospecting by electrical probing, where again $f$ represents a conductivity 
(see [4]). The second has applications in the study of ground-water flow and oil reservoirs; this problem is however not generally of the boundary measurement type (see Richter [5], Chavent [6]).

In this paper we assume that the measurements are known to arise as a response to a particular $f^{*}$, and so characterisation properties are not examined here. However, the measurements may be contaminated by noise and so computational methods of solution that are not invalidated by this possibility are examined.

To solve our inverse problem the boundary conditions are varied so giving additional information with which to reconstruct $f^{*}$. The question as to which boundary conditions can be specified so that the resulting measurements uniquely determine an arbitrary conductivity is an open one at the present time. However, this question is obviously of considerable theoretical and practical importance. Dimensionality argurnents seem to indicate at least minimum requirements to uniquely determine $f^{*}$. Take the three-dimensional case; then $f^{*}$ is a spatially varying function of three independent variables, and a single measurement on $\partial \Omega$ has only two degrees of freedom so to make the problem dimensionally deterministic the final degree of freedom for the measurements must come from known, continuously varying specified boundary conditions on $\partial \Omega$. The reasoning behind this argument is that for this problem there is no reason to expect we can uniquely reconstruct a three-dimensional vector from its two-dimensional projection. Numerous numerical experiments - that are reviewed later - seem to indicate that provided the measurements meet the conditions mentioned earlier, locally unique finite dimensional projection reconstructions of $f^{*}$ are then possible. All encompassing theoretical results however, are not available perhaps because the technique which has yielded the most results, as with many other identification problems, is analytic continuation.

Kohn and Vogelius in a series of papers [7] [8] [9] have gone some way towards answering this central uniqueness question with the result:

"If the open region $\Omega$ has a boundary $\partial \Omega \in C^{\infty}$ and $f^{*}$ is a positive piecewise real analytic function, then it is uniquely determined by knowledge of $f \frac{\partial u}{\partial \nu}$ on $\partial \Omega$ for all possible Dirichlet data $g \in H^{\frac{1}{2}}(\partial \Omega)^{\prime \prime}$.

Here $H^{\frac{1}{2}}$ denotes the Sobolev Hilbert space of distributions such that their "half" derivatives are $L^{2}(\Omega)$. We point out that this result includes practically all $f^{*}$ of computational interest and even handles $f^{*}$ having edges. This is because it is the "weak" formulation of (1.1) that is of concern here and so with the given assumptions, the solution to the direct problem is such that $u$ is at least in $H^{1}(\Omega)$. We conjecture that Kohn and Vogelius's result also applies if measurements (b) instead of (a) are used. Other authors have considered uniqueness of related identification problems but these not of so much interest to us here, as they require $f \in C^{\infty}(\Omega),[10],[11]$.

In any computational solution of the inverse problem the knowledge that the mapping from $f$ to the measurements is continuous is important. We consider application of the Newton-Kantorovich method to the aforementioned non-linear inverse problem. The Newton-Kantorovich method proceeds by local linearisation, so of immediate concern with such methods is the justification for differentability. We provide a rigorous proof of differentability and continuity of an important operator for this problem. Thereby providing the theoretical analysis necessary to justify many of the computational schemes which 
have been developed for this problem [12],,13],[14]. The reason why we have chosen the Newton-Kantorovich method is that it is a very general approach having applications to many inverse problems differing substantially from (1.1). The application of the NewtonKantorovich method for the solution of inverse problems in general has been investigated in [15], see also applications in $[19],[20],[21]$. Provided the non-linear operator admits a linearisation this method offers the following desirable additional features:

1. Quadratic convergence in the vicinity of a local solution

2. Can be made to have global convergence by incorporating line searches.

In $\S 2$ we introduce the inverse problem and discuss it's non-linear and ill-conditioned nature. We also define the Newton-Kantorovich method as applied to this problem. $§ 3$ contains our central results on the linearisation of the non-linear operator. It also contains a result ensuring a solution even if the measurements are marred by noise. In $\S 4$ we consider an important approximation to the non-linear operator, namely a particular linear approximation. $\S 5$ completes this paper by providing numerical results of our methods on a simple example.

\section{Derivation of an Iterative Scheme for Solution of the Identification Problem.}

In the sequel we consider only specified Neumann boundary conditions on the direct problem (1.1) and where measurements are made of the resulting field $U$, on $\partial \Omega$. We consider only the Neumann boundary condition for simplicity, the Dirichlet boundary condition follows our work in a similar manner.

The field $u(\underline{x})$ is a functional of the unknown function $f$ and the boundary data $g$, to make this more explicit we shall often write the field as $u(f ; g ; \underline{x})$. The direct problem is then governed by the equations

$$
\begin{gathered}
\nabla \cdot[f(\underline{x}) \nabla u(f ; g ; \underline{x})]=0, \quad \underline{x} \in \Omega \\
f(\underline{x}) \frac{\partial}{\partial \nu} u(f ; g ; \underline{x})=g(\underline{x}), \quad \underline{x} \in \partial \Omega
\end{gathered}
$$

The direct problem as it stands does not have a unique solution as constant functions are in the null space of this operator equation. To ensure the existence of a solution, the data must lie in the range of the operator, hence we have the solvability condition

$$
\int_{\partial \mathrm{n}} g(\underline{x}) d S=0 .
$$

Then to produce a unique solution, $u\left(f ; g ; \underline{x}_{0}\right)$ is specified for some $\underline{x}_{0} \in \Omega$. The energy of the solution of (2.1) is important in the sequel and is given by

$$
E_{f}(g)=\int_{\Omega} f|\nabla u|^{2} d \underline{x}
$$


To prove Fréchet differentiability in $\S 3$, we shall have to consider $f$ and $u$ being members of appropriate Banach spaces. For the inverse problem we require as little restriction on $f$ as possible, so we choose $f \in F$ where $F=\left\{f \in L^{\infty}(\Omega): \inf _{\Omega} f>0\right\}$. The weak formulation of (2.1) is then the most appropriate, and is

$$
\int_{\Omega} f \nabla u \cdot \nabla w d \underline{x}-\int_{\partial \Omega} f w g d S=0, \quad \text { for all } w \in H^{1}(\Omega) .
$$

So we shall consider $u \in H^{1}(\Omega)$, where $H^{s}(\Omega), s \geq 0$ is the Soblev space of tempered distributions, having support for all compact subsets of $\Omega$, and whose Fourier transform is square-integrable in $\mathbf{R}^{n}$ for the measure $\left(1+|\xi|^{2}\right)^{s}$, where $\xi \in \mathbf{R}^{n}$ is the Fourier transform variable. The norm is denoted by $\|\cdot\|_{s, n}$; similar conventions apply for $H^{s}(\partial \Omega), s \geq 0$. If $s<0, H^{-s}$ denotes the dual of $H^{s}$. When the $L_{\infty}(\Omega)$ norm is used, we denote it by $\|\cdot\|_{\infty, \Omega}$. The specified data $g$ will be considered to be in $H^{-1 / 2}(\partial \Omega)$, we note that measurements are often in the smoother space $H^{1 / 2}$. Another reason for considering a weak solution is that an edge condition must be imposed on $u$, when it is a classical solution, to ensure uniqueness for general $f$. This is because $f$ can have corners or edges. The edge condition which amounts to the specification of finite energy in the vicinity of the edge will be automatically satisfied when $u \in H^{1}(\Omega)$. This requirement is essential in inverse problems where there is no a priori knowledge of $f$.

The choice $f \in F$ means that the equation (2.1) will not possess a classical solution throughout $\Omega$. However a solution of the weak formulation (2.4) will automatically satisfy the classical jump conditions required across a surface, denoted by $\Gamma$, across which $f$ has a discontinuity. One of these jump conditions is

$$
\left[f \frac{\partial u}{\partial \nu}\right]=0
$$

We now define the notation used in (2.5), if $\hat{\nu}$ is a unit normal vector on a surface $\Gamma$, the difference between the values taken by the field $\phi$ on the side of $\Gamma$ towards which and away from which $\hat{\nu}$ is directed, is called the jump of $\phi$ on $\Gamma$ and is denoted by $[\phi]$. The other classical jump condition required across such a surface is $[u]=0$, that is $u \in C(\Omega)$. This will be achieved by the weak solution if $u \in H^{2}(\Omega)$ - by the Sobolev embedding theoremwhen $n=2$, or 3 . This increased regularity of the weak solution is achieved provided $\Omega$ satisfies certain smoothness properties (see [16]).

It is convenient to sometimes attach a subscript to the specified data $g_{p}$ for the problem (2.1), the associated solution then being denoted by $u_{p}$. When (2.1) is to hold for $u_{q}$ and it is multiplied by $u_{p}$ and the divergence theorem applied to the result on $\Omega$, we can show

$$
\int_{\Omega} f \nabla u_{q} \cdot \nabla u_{p} d \underline{x}=\int_{\partial \Omega} u_{p} f \frac{\partial u_{q}}{\partial \nu} d S
$$

where the right-hand-side represents the dual pairing $H^{1 / 2}(\partial \Omega) \otimes H^{-1 / 2}(\partial \Omega)$. When $p=q$ the left-hand-side represents the energy of the solution (see (2.3)). Then in particular if $f \frac{\partial u_{p}}{\partial \nu}=g_{p}$, and $p=q$ the right-hand-side is considered also as a measure of the energy in $\Omega ; f \frac{\partial u}{\partial \nu}$ being the weakly defined flux. 
If the measurements of $u$ on $\partial \Omega$ are denoted by $U$, the inverse problem can be formulated as the non-linear operator equation

$$
R(f) \equiv u\left(f ; g_{q} ; \underline{x}\right)-U\left(g_{q} ; \underline{x}\right)=0, \quad \underline{x} \in \partial \Omega .
$$

Clearly, if $f$ satisfies the conditions required in (2.1) and the measurements are exact, then the existence of a solution to (2.7) is assured. First considering $R$ as a linear operator mapping $u$ in $\Omega$ onto its boundary values on $\partial \Omega$, we see that it is a trace operator [Tréves [17], p249], hence $U \in H^{1 / 2}(\partial \Omega)$. It can be shown $R$ is a bounded continuous operator on these spaces (Connolly \& Wall [18]). It follows therefore, if the measurements $U$ are taken from the restriction of $H^{\frac{1}{2}}(\partial \Omega)$ into $H^{0}(\partial \Omega)$ and $R$ considered as $R: F(\Omega) \mapsto H^{0}(\partial \Omega)$, this map will be compact (by the Rellich embedding theorem), thereby implying that its inverse if it exists will be unbounded. The problem will therefore be ill posed in the sense of Hadamard, as the solution $f$ will not depend continuously upon the data $U$. The consequent regularisation which must be carried out will be discussed further in $\$ 3$.

To solve (2.7) for $f, R(f)$ is linearised and a series of linear subproblems is solved; this leads to the Newton-Kantorovich iterative scheme:

$$
f^{(k+1)}=f^{(k)}+s^{(k)}, \quad k=0,1,2, \ldots,
$$

given $f^{(0)}$ and where the update $s^{(k)}$ is the solution of the linear operator equation

$$
R^{\prime}\left(f^{(k)}\right) s^{(k)}=-R\left(f^{(k)}\right) \text {. }
$$

To utilise this method the Fréchet derivative of $R(f)$ with respect to $f$, denoted by $R^{\prime}$ must be known. We shall not consider the operator $R^{\prime}$ any further here as it will involve the calculation of Green's functions and this produces a method with excessive computational requirements; but see [15]. So we will now look at a method which only requires computation of the direct problem solutions.

It is important to note ( 2.7$)$ is not the only non-linear operator available for this problem, indeed any continuous linear functional of the right-hand-side is also suitable. Defining the operator

$$
Q(f)=\int_{\partial \mathrm{\Omega}} g_{p}(\underline{x})\left[u\left(f ; g_{q} ; \underline{x}\right)-U\left(g_{q} ; \underline{x}\right)\right] d S, \quad g_{p}, g_{q} \in H^{-1 / 2}(\partial \Omega),
$$

where this definition comes from the inner product of $R(f)$ with the functions $g_{p}$. Here the $g_{p}, g_{q}$ are specified boundary functions on $\partial \Omega$, and to obtain reconstructions of $f$ the $g_{p}, g_{q}$ are taken from a basis in $H^{-1 / 2}(\partial \Omega)$. When $p=q$ in (2.10) the energy of the direct problem solution (see (2.6)) is required to match that of the measurements. However we will show in $\S 4$, by example that $p \neq q$ is required to obtain reconstructions when the boundary data is taken from such a basis.

The identification problem can now be stated as the non-linear functional equation

$$
Q(f)=0,
$$

where $Q: F(\Omega) \mapsto \mathbf{R}$. In fact $Q$ can be considered as a non-linear generalised moment equation. Similar to (2.7) if $f$ satisfies the conditions required in (2.1) and the measurements are exact, then the existence of a solution to (2.11) is assured. To solve (2.11) for 
$f, Q(f)$ is linearised with the Newton-Kantorovich iterative scheme (2.8), where now the update $s^{(k)}$ is the solution of the linear operator equation

$$
Q^{\prime}\left(f^{(k)}\right) s^{(k)}=-Q\left(f^{(k)}\right)
$$

Where to utilise this method the Fréchet derivative $Q^{\prime}$ is required, the differential is

$$
Q^{\prime}(f) s=\int_{\Omega} \nabla u\left(f ; g_{p} ; \underline{x}\right) \cdot \nabla u\left(f ; g_{q} ; \underline{x}\right) s(\underline{x}) d \underline{x} .
$$

Derivation of this differential is given in the proof of Theorem 1 in $\S 3$. We note that (2.12) is a linear moment problem for the update function $s^{(k)}(\underline{x})$, where $\underline{x} \in \Omega \subset \mathbf{R}^{3}$.

Later we shall need the fact that the functional equation $(2.13)$ is a symmetric functional of $g_{p}$ and $g_{q}$ provided $U$ represents exact measurement data. This follows immediately from the observation

$$
\int_{\partial \Omega} g_{p}(\underline{x}) u\left(f ; g_{q} ; \underline{x}\right) d S=\int_{\partial \mathrm{\Omega}} g_{q}(\underline{x}) u\left(f ; g_{p} ; \underline{x}\right) d S,
$$

which is deduced from equation (2.6).

\section{Fréchet Differentiability and Considerations of Well Posedness}

Before proceeding with any computational implementation of the method advocated for the non-linear moment operator $Q(f)$ derived in the last section, we must examine the existence of the Fréchet derivative for $Q$ defined on suitable function spaces. Prior to proving differentiability we need the results of three lemmas, these show respectively

(i) Continuous dependence of the solution of the Neumann direct problem on the boundary data $g$,

(ii) Continuous dependence of $\nabla u$ on the function $f$,

(iii) $Q^{\prime}(f)$ is a bounded operator.

Lemma 1 For $f \in F(\Omega)$, the subset of positive functions from $L_{\infty}(\Omega) u \in \stackrel{\circ}{H^{1}}(\Omega)$, where $H^{1}(\Omega)$ is the quotient space of $H^{1}(\Omega)$ modulo the constant functions, and $g \in H^{-1 / 2}(\partial \Omega)$ then $u$ satisfies the well-posedness condition

$$
\|u\|_{1, \Omega} \leq C(f, \Omega)\|g\|_{-1 / 2, \partial \Omega}
$$

Proof:

Follows standard weak theory for partial differential equations - see Tréves [17].

Lemma 2 If $\nabla \cdot\left(f_{1} \nabla u_{1}\right)=0$ and $\nabla \cdot\left(f_{2} \nabla u_{2}\right)=0$, both for $\underline{x} \in \Omega$ 
(i) then

$$
\int_{\Omega} \nabla u_{1} \cdot \nabla u_{2}\left(f_{1}-f_{2}\right) d \underline{x}=\int_{\partial \Omega}\left(f_{1} u_{2} \frac{\partial u_{1}}{\partial \nu} u_{2}-f_{2} u_{1} \frac{\partial u_{2}}{\partial \nu}\right) d S
$$

(ii) If also

$$
\begin{gathered}
f_{1} \frac{\partial u_{1}}{\partial \nu}=f_{2} \frac{\partial u_{2}}{\partial \nu}, x \in \partial \Omega, \\
\frac{\left\|\nabla\left(u_{1}-u_{2}\right)\right\|_{0, \Omega}}{\left\|\nabla u_{1}\right\|_{0, \Omega}} \leq \frac{\left\|f_{1}-f_{2}\right\|_{\infty, \Omega}}{\inf _{\Omega} f_{2}},
\end{gathered}
$$

Proof:

(i) From initial assumptions

$$
\int_{\Omega} u_{1} \nabla \cdot\left(f_{2} \nabla u_{2}\right) d \underline{x}=0
$$

then utilising (A1)

$$
\int_{\Omega} f_{2} \nabla u_{1} \cdot \nabla u_{2} d \underline{x}=\int_{\partial \mathrm{\Omega}} f_{2} u_{1} \frac{\partial u_{2}}{\partial \nu} d S .
$$

Similarly one can show

$$
\int_{\Omega} f_{1} \nabla u_{1} \cdot \nabla u_{2} d \underline{x}=\int_{\partial \mathrm{\Omega}} f_{1} u_{2} \frac{\partial u_{1}}{\partial \nu} d S .
$$

By subtracting ( 3.2 ) from ( 3.3 ) we get the required result.

(ii) From the initial assumptions

$$
\nabla \cdot\left[\left(f_{1}-f_{2}\right) \nabla u_{1}\right]=\nabla \cdot\left[f_{2} \nabla\left(u_{2}-u_{1}\right)\right]
$$

and so

$$
\int_{\Omega}\left(u_{1}-u_{2}\right) \nabla \cdot\left[\left(f_{1}-f_{2}\right) \nabla u_{1}\right] d \underline{x}=\int_{\Omega}\left(f_{1}-f_{2}\right) \nabla \cdot\left[f_{2} \nabla\left(u_{2}-u_{1}\right)\right] d \underline{x} .
$$

Applications of (A2) to ( 3.4) and utilising assumptions for part (ii) shows

$$
\int_{\Omega}\left(f_{1}-f_{2}\right) \nabla u_{1} \cdot \nabla\left(u_{1}-u_{2}\right) d \underline{x}=\int_{\Omega} f_{2}\left|\nabla\left(u_{1}-u_{2}\right)\right|^{2} d \underline{x} .
$$

The Cauchy-Schwarz inequality now gives

$$
\left\|\left(f_{1}-f_{2}\right) \nabla u_{1}\right\|_{0, \Omega} \geq \inf _{\Omega} f_{2}\left\|\nabla\left(u_{1}-u_{2}\right)\right\|_{0, \Omega}^{2}
$$

so that

$$
\left\|f_{1}-f_{2}\right\|_{\infty, \Omega} \quad\left\|u_{1}\right\|_{0, \Omega} \geq \inf _{\Omega} f_{2}\left\|\nabla\left(u_{1}-u_{2}\right)\right\|_{0, \Omega}
$$

as required. The result (ii) follows Richter [5], we have included this proof for completeness. 
Lemma 3 The mapping $Q^{\prime}(f): F(\Omega) \mapsto \mathbf{R}$ is a continuous linear functional.

Proof: From ( 2.13)

$$
Q^{\prime}(f) s=\int_{\Omega} \nabla u\left(f ; g_{p}\right) \cdot \nabla u\left(f ; g_{q}\right) s(\underline{x}) d \underline{x}
$$

and so

$$
\left|Q^{\prime}(f) s\right| \leq\left[\int_{\Omega}\left|\nabla u\left(f, g_{p}\right) \cdot \nabla u\left(f ; g_{q}\right)\right| d \underline{x}\right]\|s\|_{\infty, \Omega}
$$

and by the Cauchy-Schwartz inequality

$$
\left|Q^{\prime}(f) s\right| \leq\left\|\nabla u\left(f ; g_{p}\right)\right\|_{0, \Omega}\left\|\nabla u\left(f ; g_{q}\right)\right\|_{0, \Omega}\|s\|_{\infty, \Omega} .
$$

From Lemma 1 it follows $\|\nabla u(f ; g)\|_{0, \Omega} \leq C\left\|_{g}\right\|_{-1 / 2, \partial \Omega}$ for any boundary data $g \in H^{-1 / 2}(\partial \Omega)$ then

$$
\left|Q^{\prime}(f) s\right| \leq C\left(\Omega, g_{p}, g_{q}\right)\|s\|_{\infty, \Omega},
$$

It follows $Q^{\prime}$ is a bounded linear operator, and hence continuous. Its norm - an operator norm - is bounded by the infimum of $C$ for fixed $g_{p}, g_{q}$, that is

$$
\left\|Q^{\prime}(f)\right\|=\inf C\left(f, \Omega, g_{p}, g_{q}\right) .
$$

A norm independent of $g_{p}, g_{q}$ can be found by finding the infimum of $C$ which is also over the $g$, for $\left\|g_{p}\right\|_{-1 / 2, \partial \Omega}=\left\|g_{q}\right\|_{-1 / 2, a n}=1$. We shall use this norm on the operator $Q$ and its derivatives in the sequel.

We can now state the differentiability result.

\section{Theorem 1 .}

If $\Omega$ is a bounded domain with a $C^{1}$ boundary and with Neumann boundary conditions such that $g_{p}, g_{q} \in H^{-1 / 2}(\partial \Omega)$ then the non-linear functional $Q: F \mapsto \mathbf{R}$ given by (2.11) is Fréchet differentiable with Fréchet differential given by (2.13).

Proof: Define

$$
\begin{aligned}
w(f ; s)= & Q(f+s)-Q(f)-Q^{\prime}(f) s \\
= & \int_{\partial \Omega} g_{q}\left[u\left(f+s ; g_{p}\right)-U\left(g_{p}\right)\right] d S \\
& -\int_{\partial \Omega} g_{q}\left[u\left(f ; g_{p}\right)-U\left(g_{p}\right) d S\right. \\
& -\int_{\Omega} \nabla u\left(f ; g_{p}\right) \cdot \nabla u\left(f ; g_{q}\right) s d \underline{x} .
\end{aligned}
$$

Considering the first two integrals on the right-hand-side of (3.6) use of the symmetry property (2.14) shows

$$
\begin{aligned}
\int_{\partial \Omega} g_{q}\left[u\left(f+s ; g_{p}\right)-u\left(f ; g_{p}\right)\right] d S & =\int_{\partial \Omega}\left[g_{p} u\left(f+s ; g_{q}\right)-g_{q} u\left(f ; g_{p}\right)\right] d S \\
& =-\int_{\Omega} \nabla u\left(f+s ; g_{q}\right) \cdot \nabla u\left(f, g_{p}\right) s d \underline{x}
\end{aligned}
$$


where we have used Lemma $1(i)$. It follows

$$
w(f ; s)=-\int_{\Omega} \nabla u\left(f, g_{p}\right) \cdot \nabla\left[u\left(f+s ; g_{q}\right)-u\left(f, g_{q}\right)\right] s d \underline{x}
$$

and

$$
|w(f ; s)| \leq\left[\int_{\Omega}\left|\nabla u\left(f, g_{p}\right) \cdot \nabla\left(u\left(f+s ; g_{q}\right)+u\left(f, g_{q}\right)\right)\right| d \underline{x}\right]\|s\|_{\infty, \Omega} .
$$

By using the Schwartz inequality on this equation we get

$$
|w(f ; s)| \leq\left\|\nabla u\left(f, g_{p}\right)\right\|_{0, \Omega}\left\|\nabla\left[u\left(f+s ; g_{q}\right)-u\left(f, g_{q}\right)\right]\right\|_{0, \Omega}\|s\|_{\infty, \Omega}
$$

and Lemma 1(ii) then shows

$$
\left\|\nabla\left[u\left(f+s ; g_{q}\right)-u\left(f ; g_{q}\right)\right]\right\|_{0, \Omega} \leq\|s\|_{\infty, \Omega}\left\|\nabla u\left(f+s ; g_{q}\right)\right\|_{0, \Omega} / \inf _{\Omega} f .
$$

The quantity

$$
\frac{|w(f ; s)|}{\|s\|_{\infty, \Omega}}=\left\|\nabla u\left(f, g_{p}\right)\right\|_{0, \Omega}\left\|\nabla u\left(f+s ; g_{q}\right)\right\|_{0, \Omega}\|s\|_{\infty, \Omega} / \inf _{\Omega} f
$$

gives

$$
\lim _{\|s\|_{\infty, \Omega \rightarrow 0}} \frac{\|w(f ; s)\|}{\|s\|_{\infty, \Omega}}=0,
$$

where use is made of Lemma 1. The norm on $w$ is as given in (3.5). This result together with Lemma 3 gives the required result.

It follows directly from theorem 1 , the operator $Q$ is continuous with respect to $f$.

In the presence of measurement noise we generally have

$$
U(g ; \underline{x}) \neq u\left(f^{*} ; g ; \underline{x}\right), \quad \underline{x} \in \partial \Omega,
$$

and so a solution of our operator equations may not exist. This is particularly true in the case of the operator equation (2.11), as it is a compact operator if considered as $Q: F \mapsto H^{0}(\partial \Omega)$. This will mean its inverse will be unbounded and its range will not be dense in $H^{0}(\partial \Omega)$. In order to guarantee the existence of a solution we use a priori information on the smoothness of the solution required to reformulate the inverse problem as a minimisation over a compact set (see Tikhonov and Arsenin[22]). This constitutes a regularisation technique.

When $g_{p}, g_{q}$ are chosen from a basis set in $H^{0}(\partial \Omega)$ with unit norm, we can write the operator $Q$ as $Q\left(f ; g_{p} ; g_{q}\right)$ and could consider the norm for fixed $f$,

$$
\|Q(f)\|=\sup _{g_{p}, g_{q} \in G}\left|Q\left(f ; g_{p} ; g_{q}\right)\right|
$$

where $G=\left\{g \in H^{-1 / 2}(\partial \Omega):\|g\|=1\right\}$. Then we can pose the minimisation problem

$$
\min _{f \in F_{\mathbf{0}}}\|Q(f)\|^{2},
$$


where $F_{0}$ is a compact subset of $F$. A norm more computationally convenient than (3.7) can be found by restricting the boundary data to a $\mathrm{N}$-dimensional subset of $H^{0}(\partial \Omega)$. Then we pose the minimisation problem as (3.8), but with the norm now being the Frobenius norm

$$
\|Q(f)\|_{F}=\left\{\sum_{p, q}^{N} \mid Q\left(f ; g_{p} ;\left.g_{q}\right|^{2}\right\}^{1 / 2} .\right.
$$

With the problem now set as a minimisation the following result can be obtained.

Theorem 2 There exists a solution of the minimisation problem (3.8) provided $F_{0}$ is compact and the conditions of Theorem 1 hold.

Proof: From Theorem $1 Q(f)$ is Fréchet differentiable and so is a continuous operator. The norm in (3.8) is continuous hence the theorem follows from the compactness of $F_{0}$.

One possible choice for $F_{0}$ suitable for computational purposes is

$$
F_{0}=\left\{f \in H^{m}(\Omega):\|f\| \leq M, f \geq c>0\right\} .
$$

The constants $M$ and $c$ are given a priori and the compactness of $F_{0}$ follows from the compactness of the embedding $H^{m}(\Omega) \rightarrow L^{\infty}(\Omega)$; from Adams [23])this would require $m=2$ if $f$ is 2 or 3 dimensional. If this choice of $F_{0}$ were to be used the resulting reconstructions of a discontinuous $f$ would the be smoothed. However if discontinuous reconstructions are desired the solution set $F_{0}$ could be chosen a finite dimensional subspace of $L^{\infty}(\Omega)$, such as the splines of degree zero; if we add the requirement $\|f\|_{\infty} \leq M$, then $F_{0}$ has the required compactness property. We shall use such a solution set in $\S 5$.

However the solution of the minimisation problem (3.8), say $\hat{f}$, may not be close to the $f^{*}$. What one requires is that

$$
\left\|\hat{f}-f^{*}\right\|_{\infty, \Omega} \leq \gamma\left\|u\left(f^{*} ; g ; \underline{x}\right)-U(g ; \underline{x})\right\|, \quad \underline{x} \in \partial \Omega,
$$

and $\gamma$ is known. When such inequalities are found for this inverse problem, it will be well understood. Some results of this kind are available for other inverse problems $([24],\{25],[26])$.

It should be noted that computationally the problem is not solved as a full minimisation problem which would require estimations of second derivatives. Instead ( 3.8$)$ is used to solve ( 2.11) with the Gauss-Newton method. With this approach ( 2.12) together with ( 2.8) is used, but the update equation( 2.12 ) is solved by a least-squares approach. This enables the number of measurements to be overdetermined; a desirable feature with measurement noise. A line search is usually incorporated to determine the magnitude of $s^{(k)}$ (see for example [27]).

\section{The Linearised Problem}

If an approximation denoted by $f^{0}$ is known to the solution $f^{*}$, in the non-linear problem (2.11) such that $\left\|f^{*}-f^{0}\right\|_{\infty, \Omega}$ is small enough, then as

$$
\left\|Q\left(f^{*}\right)-\left[Q\left(f^{0}\right)+Q^{\prime}\left(f^{0}\right) s\right]\right\|=o\left(\left\|f^{*}-f^{0}\right\|\right)
$$


as $f^{0} \rightarrow f^{*}$ and where $s=f-f^{0}$. Fréchet differentiability of $Q$, as shown in theorem 1 , ensures (4.1) is valid. The equation (4.1) implies the linearisation of $Q$, namely the term in square brackets inside the norm symbol on the right-hand-side of equation (4.1), is a good approximation to $Q$ near $f^{*}$. The solution of this linearised inverse problem

$$
Q^{\prime}\left(f^{0}\right) s=-Q\left(f^{0}\right)
$$

that is $f^{(1)}=s+f^{0}$, may then be expected to provide a good approximation to $f^{*}$, and some numerical results we present in $\S 5$ confirm this.

The linear versions of several inverse problems involving the wave equation are treated in Cohen and Bleistein [28]. They use the well known Born approximation to linearise their problems.

Calderon [29] has shown that there is a unique solution to the inverse problem which is linearised about an $f$ constant over $\Omega$ given knowledge of the energy $E_{f}$ from all possible Dirichlet boundary conditions. He did this by showing that the Fréchet differential of the map from $f$ to $E_{f}$ is injective, for $f$ a constant. In this paper he also gives a method for approximating $f$, when $f$ is close to constant.

We are interested here however, in the practical situation where independent boundary data are chosen from an $\mathrm{N}$-dimensional subspace of $H^{0}(\partial \Omega)$, for reconstruction of nonsmooth $f$. When $n=2$, use of the energy will only enable a one dimensional projection of the two-dimensional $f$ to be reconstructed. We shall show in the sequel that for the case when $n=2$ and $\Omega$ is a circular domain, knowledge of the energy can only be used to reconstruct radially symmetric $f$ 's. But by utilising the quantities given by the righthand-side of (2.6), when $p$ is not always equal to $q$, we shall show that a two-dimensional $f$ can be reconstructed. It should be noted when using the known value of the energy it corresponds to using only the quantities when $p=q$. We shall also show that there is a unique solution to this inverse problem linearised about an $f$ constant over $\Omega$. This shows that our approach leads to at least a locally unique solution. Our method leads to a set of uncoupled one-dimensional moment problems which can be solved with computational efficiency.

We now discuss the computational method of solving the linearised problem (4.2) for a particular problem geometry, when $n=2$. Take $\Omega$ to be the open region bounded by a circle centred on the origin and of radius unity. We set up a circular-polar coordinate system $(r, \theta)$ at this origin. This in fact involves no loss in generality, as by a partition of unity, any other open simply connected two-dimensional region can be mapped into this circle. As the specified data is to be independent, and to come from an $2 N+1$-dimensional subspace of $H^{0}(\partial \Omega)$ it is computationally convenient to take them from the trigonometric

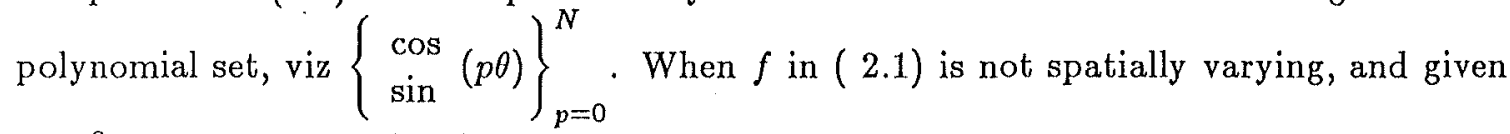
by $f^{0}$, the solution of $(2.1)$ is

$$
\stackrel{e}{u_{p}^{o}}=r^{p} \underset{\sin }{\cos }(p \theta) / f^{0}+\text { constant }
$$

where the superscripts e,o attached to $u$ are taken to mean that the even and odd trigonometric polynomials are respectively to be used on the right-hand-side of (4.3). The even 
or odd trigonometric polynomials are to be used depending upon whether the boundary data $g_{p}$ is of the even or odd type, respectively.

We then resolve the difference between the field $u_{p}(f ; \underline{x})$ and the measured field $U_{p}(\underline{x})$ on $\partial \Omega$ into the Fourier amplitudes $A_{p, m}\left(f^{*}, f^{0}\right)$ and $B_{p, m}\left(f^{*}, f^{0}\right)$, so that

$$
u_{p}\left(f^{0} ; \underline{x}\right)-U_{p}(\underline{x})=\sum_{m=0}^{\infty} \epsilon_{m}\left[A_{p, m} \cos (m \theta)+B_{p, m} \sin (m \theta)\right], \quad \underline{x} \in \partial \Omega,
$$

where $\epsilon_{m}$ is the Neumann factor. We note $B_{p, 0} \equiv 0$ throughout the sequel and we have dropped the arguments $f^{*}, f^{0}$ from the terms $A_{p, m}, B_{p, m}$. Use of (4.3), (4.4) and (2.13) in (4.2) then shows

$$
\int_{0}^{2 \pi} \int_{0}^{1} r^{p+q-1} \sin [(p-q) \theta] s(r, \theta) d r d \theta=\pi\left(f^{0}\right)^{2}+A_{p, q}(f), \quad 0 \leq p, q<\infty
$$

We note that only the equations $p \geq q$ are necessary in (4.5) because of (2.14). To solve this linear system of moment equations set

$$
s^{(0)}(r, \theta)=\sum_{m=0}^{N} \epsilon_{m}\left[a_{m}(r) \cos (m \theta)+b_{m}(r) \sin (m \theta)\right], \quad 0 \leq r \leq 1,0 \leq \theta \leq 2 \pi,
$$

and substitute this in (4.5), to give the dual uncoupled one-dimensional moment system

$$
\int_{0}^{1} r^{n+2 q-1} \frac{a_{n}(r)}{b_{n}(r)} \text { d } d r=\left(f^{0}\right)^{2} \begin{aligned}
& A_{n+q, q} \\
& -B_{n+q, q}
\end{aligned}, \quad 0 \leq n, q \leq N
$$

where we have set $n=p-q$. It is now clear that for the linearised problem (4.2), the leading diagonal of the matrix $A_{p, q}$ is used to reconstruct the radial part $a_{0}(r)$ of $s^{(0)}$, the subdiagonal (or super-diagonal) to reconstruct $a_{1}(r)$ and so forth. The matrix $B_{p, q}$ and the elements $b_{n}(r)$ are related in a similar manner.

As $L^{\infty} \subset L^{2}$, if we prove uniqueness of the moment problems in $L^{2}$ it follows the solution in $L^{\infty}$ is also unique. So by considering $(4.6)$ to have solutions in $L^{2}(0,1)$, then these solutions must be unique. This follows as the functions $\left\{r^{n+2 q+1}\right\}=\left\{r^{n+1}, r^{n+3}, r^{n+5}, \ldots\right\}$ are complete in $L^{2}(0,1)$ from the Muntz closure theorem (see [30], p267). This however does not give the existence of a solution of the moment problems (4.6), but we do have the existence of a solution to the inverse problem as a whole, when the measurements arise in response to $f^{*}$ and do not contain noise.

\section{$5 \quad$ Numerical Solution of a Radially Symmetric Inverse Problem}

We have developed in $\S 4$ a closed form expression for the Fréchet differential when $f=f^{0}$, namely (4.5). This can be utilised when solving the non-linear problem (2.1) when the modified form of the Newton-Kantorovich method is used. In this section we shall apply this method to obtain some numerical results from the reconstruction of several radially 


\begin{tabular}{|c|c|c|}
\hline $\mathrm{a}$ & number of iterations & $\left\|f^{*}-f^{(k)}\right\|_{\infty}$ \\
\hline .1 & 1 & 0.010 \\
.5 & 8 & 0.001 \\
1 & 9 & 0.031 \\
-.25 & 5 & 0.008 \\
\hline
\end{tabular}

Table 1: Results of numerical computation with the modified Newton-Kantorovich method.

symmetric $f^{*}(r)$ on a circle of unity radius, from boundary measurements. We chose to reconstruct discontinuous $f$-noting we have proved Fréchet differentiability for $f \in L^{\infty}(\Omega)$ and positive.

The modified Newton-Kantorovich scheme we use to solve (2.11) is

$$
Q^{\prime}\left(f^{0}\right) s^{(k)}=-Q\left(f^{(k)}\right), \quad f^{(k+1)}=f^{(k)}+s^{(k)},
$$

given some initial constant estimate $f^{0}$. We see the Fréchet derivative is left fixed as at the first iteration and is not updated. Thus we use the closed form Fréchet differential (4.5) in our scheme, thereby simplifying the computational complexity considerably. We stress we are not advocating this as an approach to be taken in general, but here it enables us to illustrate the computational properties of the Newton-Kantorovich scheme with simple calculations.

Other authors [31],[32],[33] have considered inverse problems involving reconstruction of one-dimensional $f$. However the methods they use are not as powerful as ours in that either, they will not handle discontinuities in $f$, or the methods cannot be generalised to n-dimensional $f$.

As the radially symmetric $f^{*}$ is clearly even in $\theta$, only the even moment equations in (4.6) are needed, where now the $a_{n}$ and $A_{p, q}$ have an iteration number $k$ appended as a superscript. It suffices because of the discussion after equation (4.6) to set $n=0$. In order to use the Fréchet differential ( 4.6) it is necessary to approximate the $a_{n}^{(k)}(r)$ by piecewise constant functions. This is of no numerical disadvantage, as splines of degree zero are the appropriate polynomials to use for the approximation of the discontinuous function $f^{*}$. We chose for the $f^{*}$ to be reconstructed the step function

$$
f^{*}(r)= \begin{cases}1+a, & 0 \leq r \leq 1 / 2 \\ 1, & 1 / 2<r \leq 1\end{cases}
$$

for various values of the constant $a$. Table 1 summarises our numerical results when ten uniform intervals on $[0,1]$ were used for the piecewise constant basis.

In all cases tested the condition number of the linear algebraic system of equations to be solved was of the order $10^{5}$. The order of convergence of our method was linear; as expected for the modified Newton-Kantorovich method. Convergence was monotone for all positive values of $a$ tested, but changed to oscillatory for $a<0$. In fact convergence was difficult to achieve for negative $a$ and the method failed to converge for all $a<-.3$, illustrating that the problem was too non-linear for the initial linear approximation to be 


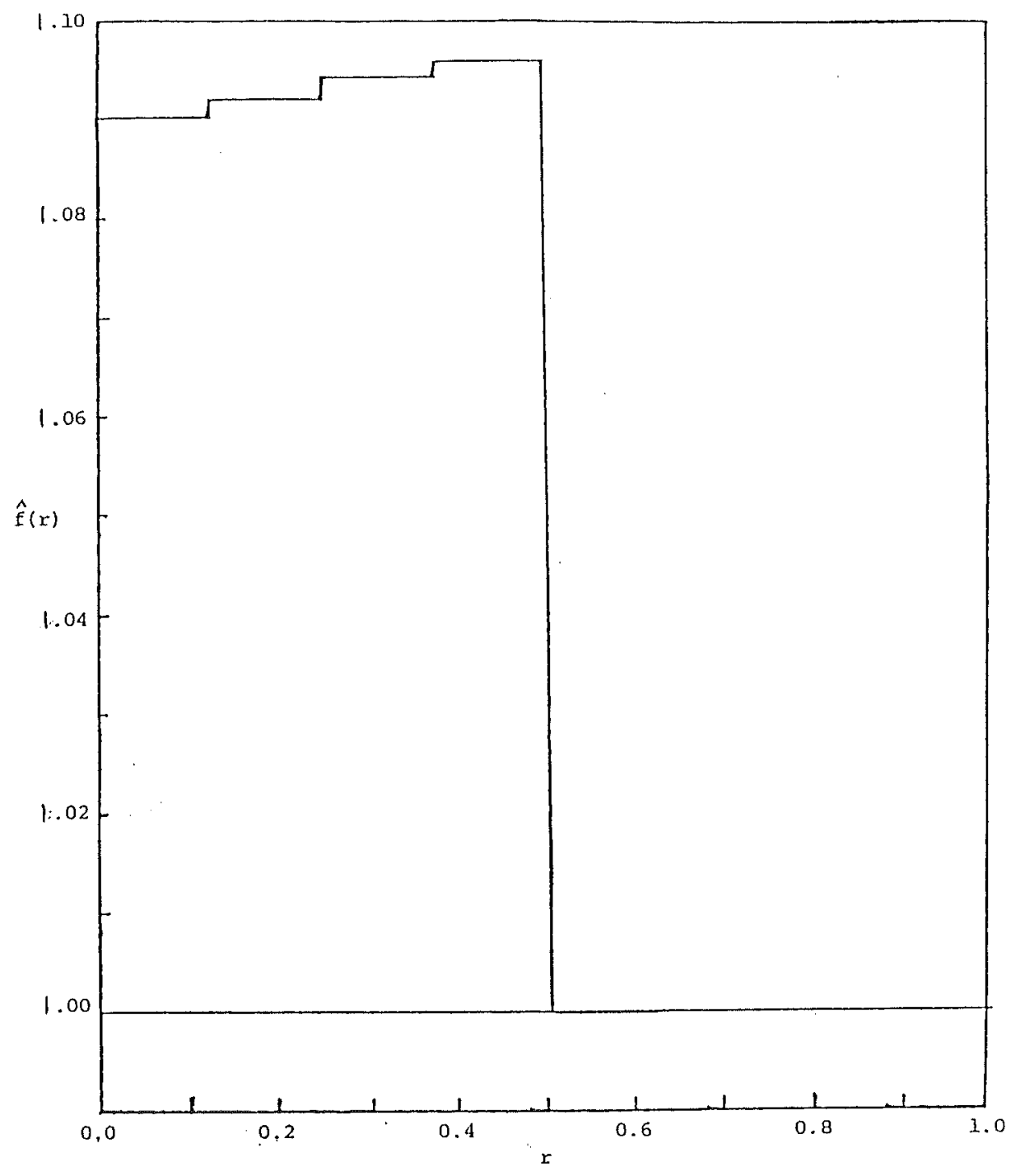

Figure 1: Solution $\hat{f}$ from linearised problem, when $a=0.1$ in (5.1).

satisfactory for these values of $a$. We started off with an initial approximation $f^{(0)}=1 \mathrm{in}$ all cases. We stress again, that using a full Newton-Kantorovich implementation, where the Fréchet derivative is updated, convergence for these more difficult cases would be expected.

For $a=.1$ we illustrate in Figure 1 the solution obtained after one iteration, that is just the solution of the linearised problem as given in (4.2). This figure illustrates how for small perturbations, the linearised model can give remarkably good results.

\section{Appendix}

Use has been made of the following generalisation of Green's theorems (see [31], p127). The first theorem: If $\Omega$ is regular bounded region, and $u_{1}, f \in C^{1}(\Omega), u_{2} \in C^{2}(\Omega)$ then

$$
\int_{\Omega} u_{1} \nabla \cdot\left(f \nabla u_{2}\right) d \underline{x}=-\int_{\Omega} f \nabla u_{1} \cdot \nabla u_{2} d \underline{x}+\int_{\partial \Omega} f u_{1} \frac{\partial u_{2}}{\partial \nu} d S .
$$

When $\Omega$ is a regular its boundary $\partial \Omega$ is a piecewise smooth orientable surface. A surface $\partial \Omega$ is smooth if it has a unique normal whose direction depends continuously on points of 
$\partial \Omega$. The second theorem:

$$
\int_{\Omega}\left[u_{1} \nabla \cdot\left(f \nabla u_{2}\right)-u_{2} \nabla \cdot\left(f \nabla u_{1}\right)\right] d \underline{x}=\int_{\partial \Omega} f\left[u_{1} \frac{\partial u_{2}}{\partial \nu}-u_{2} \frac{\partial u_{1}}{\partial \nu}\right] d S .
$$

\section{References}

(1) Bates, R.H.T., McKinnon, G.C. and Segar, A.D., 1980, A limitation on systems for imaging electrical conductivity distributions, IEEE Trans. Biomedical Eng., 19, pp 418-420.

[2] Price, L.R., 1979, Electrical impedance computed tomography (ict): a new c.t. imaging technique, IEEE Trans. Nuclear Sci., 26, pp 2736-2739.

[3] Barber, D.C., and Brown, B.H., 1984, Applied potential tomography, J. Phys: E, 17, pp 723-732.

[4] Dines, K.A., and Lyttle, R.J., 1981, Analysis of electrical conductivity imaging, Geophysics, 46, pp 1025-1036.

[5] Richter, G.R., 1981, An inverse problem for the steady state diffusion equation, SIAM J. Appl. Math., 41, pp 210-221.

[6] Chavent, G., 1973, Identification of distributed parameters, pp 649-660 in Eykhoff, P. editor, Identification and System Parameter Estimation, Proc. third IFA Symp., North-Holland .

[7] Kohn, R.V., and Vogelius, M., 1984, Determining conductivity by boundary measurements, Comm. Pure Appl. Math, 37, pp 289-298.

[8] Kohn, R.V., and Vogelius, M., 1984, Identification of an unknown conductivity by measurements at the boundary, in McLaughlin, D.W., editor, Inverse Problems, SIAM Proc. 19, pp 113-123.

[9] Kohn, R.V., and Vogelius, M., 1985, Determining conductivity by boundary measurements II, interior results, Comm. Pure Appl. Math., 38, pp 643-667.

[10] Sylvester, J., and Uhlmann, G., 1986, A uniqueness theorem for an inverse boundary value problem in electrical prospection, Comm. Pure Appl. Math. 39, pp 91-112.

[11] Sylvester, J., and Uhlmann, G., 1986, A global uniqueness therem for an inverse boundary value problem, Annals of Math., to appear.

[12] Murai, T. and Kagawa, Y., 1985 Electrical impedance computed tomography based on a finite element model, IEEE Trans. Biomedical Eng., 43, pp 788-803.

[13] Yorkey, T.J., and Webster, J.G., 1987 A comparison of impedance tomographic reconstruction algorithms, Clin. Phys. Physiol. Meas., Special Issue, 8, pp 55-62. 
[14] Breckon, W.R. and Pidcock, M.K., 1987, Mathematical aspects of the impedance imaging, Clin. Phys. Physiol. Meas., Special Issue, 8, pp 77-84.

[15] Connolly, T.J., Wall, D.J.N., and Bates, R.H.T., 1985, Inverse problems and the Newton-Kantorovich method, pp 30-34, in Bates, R.H.T. and Devaney, A.J., editors, Inverse Optics II, Proc. of the International Soc. for Optical Engineering, 558.

[16] Gilbarg, D., Trudinger, N.S., 1983, Elliptic Partial Differential equations of Second Order, Springer-Verlag, Berlin.

[17] Tréves, F., 1975, Basic Linear Partial Differential Equations, Academic Press, New York.

[18] Connolly, T.J., and Wall, D.J.N., 1987, Fréchet differentiability of non-linear operators in inverse problems, to appear.

[19] Chen, Y.M., and Lui, J.Q., 1982, A numerical algorithm for solving inverse problems of two-dimensional wave equations, J. Comp. Phys., 50, pp 193-208.

[20] Lui, J.Q., and Chen, Y.M., 1984, An iterative algorithm for solving inverse problems of two-dimensional diffusion equations, SIAM J. Sci. Stat. Comp., 5, pp 255-269.

[21] Roger, A., 1981, Newton-Kantorovich algorithm applied to an electromagnetic inverse problem, IEEE Trans. on Antennas and Propag., em 29, pp232-238.

[22] Tikhonov, A.N. and Arsenin, V.Y., 1977, Solutions of Ill-posed problems, V.H. Winston, Washington D.C.

[23] Adams, R.A., 1975, Sobolev Spaces, Academic Press, New York.

[24] Colton,D., and Kress, R., 1983, Integral Equation Methods in Scattering Theory, John Wiley, New York.

[25] Kravais, C. and Seinfeld, J.H., 1985, Identification of parameter systems by regularization, SIAM J. Control Opt., 23, pp 217-241.

[26] Betero, M., De Mol, C., and Viano, G.A., 1979, The stablity of inverse problems, pp 116-124, in Baltes, H.P, editor, Inverse Scattering Problems in Optics, SpringerVerlag, Berlin.

[27] Dennis, J.E., Schnabel, R.B., 1983, Numerical Methods for Unconstrained Optimization and Nonlinear Equations, Prentice-Hall, Englewood Cliffs.

[28] Cohen, J.K. and Bleistein, N. 1977, An inverse method for determining small variations in propagation speed, SIAM J. Appl. Math., 32, pp 784-799.

[29] Calderon, A.P., 1980, On an inverse boundary value problem, Seminar on Numerical Analysis and its Applications to Continuum Mechanics, Soc. Brasileira de Matematica, Rio de Janeiro, pp 65-73. 
[30] Davis, P., 1963, Interpolation and Approximation, Blaisdell, New York.

[31] Coen, S., and Yu, M.W-H., 1981, The inverse problem of the direct current conductivity profile of a layered earth, Geophysics, 46, pp 1702-1713.

[32] Langer, R.E., 1933, An inverse problem in differential equations, Bull. Amer. Math. Soc., 39, pp 814-820.

[33] Langer, R.E., 1936, On the determination of earth conductivity from observed surface potentials, Bull. Amer. Math. Soc., 10, pp 747-754.

[34] Williams, W.E., 1980, Partial Differential Equations, Clarendon Press, Oxford.

[35] Wall, D.J.N., Yeo, T.S. and Bates, R.H.T., 1985, Inverse scattering and the null field method, pp 25-29, in Bates, R.H.T. and Devaney, A.J., editors, Inverse Optics II, Proc. of the International Soc. for Optical Engineering, 558. 